\title{
Effect of Different Types of Fabricated Anti-scatter Grids in Reducing Backscattered Radiation
}

\author{
Abdullah Taher Naji ${ }^{1,2^{*}}$ and Mohamad Suhaimi Jaafar ${ }^{1}$ \\ ${ }^{1}$ School of Physics, Universiti Sains Malaysia, \\ 11800 USM Pulau Pinang, Malaysia \\ ${ }^{2}$ Health Science Department, Faculty of Medicine and Health Science, \\ University of Science and Technology, 00967 UST Sixty Street, Sana'a, Yemen \\ "Corresponding author: ataher8383@yahoo.com
}

Published online: 15 August 2017

To cite this article: Naji, A. T. \& Jaafar, M. S. (2017). Effect of different types of fabricated anti-scatter grids in reducing backscattered radiation. J. Phys. Sci., 28(2), 105-114, https://doi.org/10.21315/jps2017.28.2.7

To link to this article: https://doi.org/10.21315/jps2017.28.2.7

\begin{abstract}
This study aims to reduce backscattered radiation inside an X-ray room during X-ray imaging. A number of anti-scatter grids with different geometrical designs have been fabricated from iron steel and aluminium independently because of their relatively better abilities to reduce backscattered radiation compared to other materials, to elucidate the backscattered radiation reduction capabilities of fabricated grids utilising different X-ray machines and several X-ray energies. Crossed iron steel grid exhibited the best backscattered radiation reduction of up to $49.24 \%$ under low energy of radiation exposure $(50 \mathrm{kVp})$.
\end{abstract}

Keywords: Anti-scatter grid, X-ray, backscattered radiation, reduction, crossed iron steel

\section{INTRODUCTION}

Scattered X-ray is an intricate phenomenon that can cause significant degradation of image quality, mainly in terms of contrast and resolution, and contribute to image noise. In addition, it generates additional unwanted radiation dose to patients and personnel in an X-ray room. ${ }^{1,2}$ The problem of scattered radiation exposure to medical personnel during radiological operation is imperative to pay attention due to the position of the workers and patients in relation to the radiation source, and the long period of X-ray exposure. Hence, scattered radiation is considered the main source of the occupational radiation exposure. ${ }^{3,4}$ Therefore, there is a need to 
reduce backscattered radiation to protect patients and workers in an X-ray room from unnecessary radiation exposure.

Scattered radiation can be categorised based on the direction of secondary photons. Backscattered radiation consists of photons that collide with an object and are reflected back at different backscattering angles. When the photons are reflected back with scattering angle greater than $90^{\circ}$ (at various angles between $90^{\circ}$ and $180^{\circ}$ ) with the incident photons, it is referred to as backscattered radiation. On the other hand, sidescattered radiation occurs when the photons scatter to the side or changed its trajectory by $90^{\circ}$ from the initial photon beam direction. ${ }^{5,6}$

The effect of backscattered radiation on image receptor and patient is significant because of the short distance from the source of backscattered radiation to patient's table, floor, standing bucky and wall. ${ }^{7,8}$ In diagnostic radiography, backscattered radiation is responsible for the cassette-hinge image which is sometimes seen on radiograph, even though the hinge is on the back side of the cassette. ${ }^{9}$ In such situation, the X-ray photons have backscattered from the wall of the room or the imaging table. ${ }^{4}$

When attenuated photons are transmitted through an exposed target and collide with patient's table or wall bucky, some of the backscattered photons reach the film screen or detectors, possibly affecting the imaging system and initiating the presence of redundant radiation dose in the X-ray room. Therefore, scattered radiation photons (either sidescattered or backscattered) are constantly taken into consideration when producing a radiograph due to its effect on the image quality and exposure of medical personnel to the X-ray. ${ }^{10,11}$

This study will offer a novel practical method to decrease backscattered radiation exposure for patients, medical personnel, public and image's receptor. The materials utilised in this work are economical and readily available. They can also easily be shaped and fabricated into different designs of anti-scatter grids.

\section{EXPERIMENTAL}

\subsection{Materials}

First, X-ray mass attenuation coefficients of $1 \mathrm{~mm}$ thickness for different metals were investigated to determine the capability of available materials in attenuating incident X-ray as illustrated in Figure 1. As illustrated in the figure, lead possesses higher value of mass attenuation coefficients compared to aluminium, while those 
of iron and copper lie in between them. This observation is attributed to the effect of mass density and atomic number on the values of mass attenuation coefficient for materials.

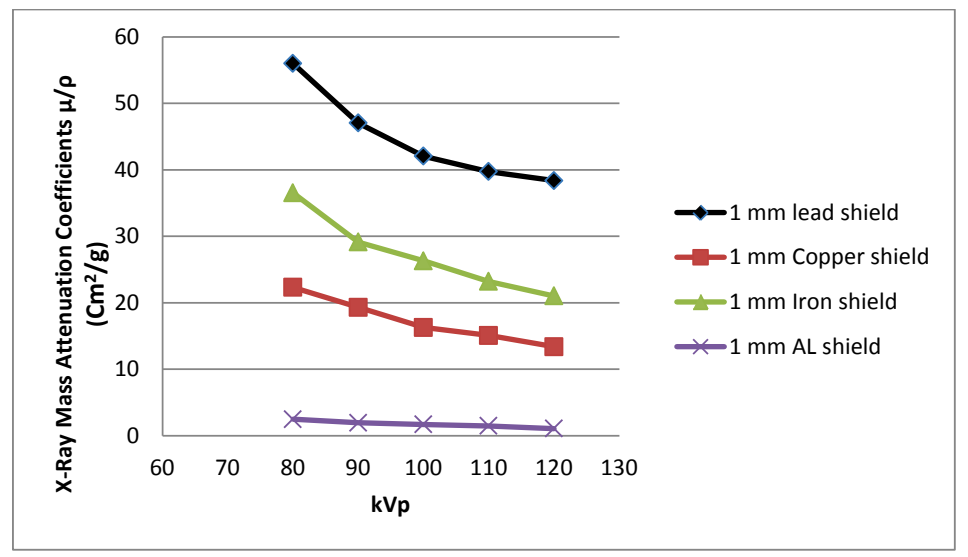

Figure 1: Mass attenuation coefficient for different metals with $1 \mathrm{~mm}$ thickness.

Then, the anti-scatter grids were fabricated from different materials (iron steel and aluminium) which are readily available and are relatively low cost, as well as its properties in reflecting less backscatter radiation, and can be shaped into different geometrical designs (crossed and linear). The grid's construction permits controlling of the space between strips by ejecting and inserting the moveable strips. Figure 2 illustrates the geometrical designs of the constructed anti-backscattered $\mathrm{X}$-ray grids.

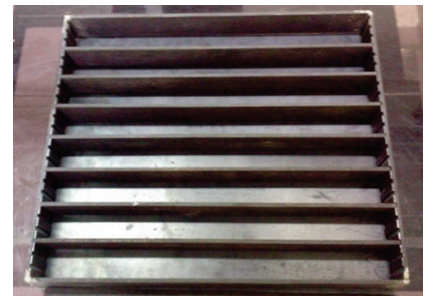

Linear Grid

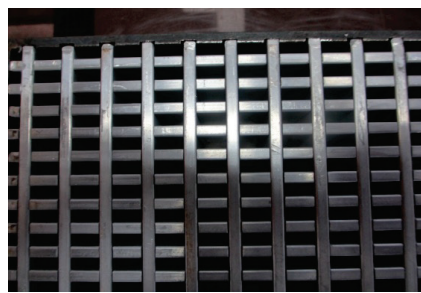

Crossed Grid

Figure 2: Geometrical designs of fabricated anti-scatter grids. 


\subsection{Methods}

These grids were examined to evaluate their effectiveness in reducing the backscattered radiation. The fabricated grids were placed on an X-ray table under image receptor and an exposed object. The distance between X-ray machine source and surface of imaged phantom (SSD) is fixed at $85 \mathrm{~cm}$ for all sittings. The detectors (dosimeters) were positioned at a level between X-ray source and patient's table at backscattered angle $45^{\circ}$ with the central ray of incident X-ray beam $\left(135^{\circ}\right.$ between the incident and the backscattering radiation). The dosimeters were positioned outside the exposed area to measure backscattered radiation dose only.

\section{RESULTS AND DISCUSSION}

The dose of backscattered radiation for different X-ray units and the backscattered radiation reduction capabilities of several anti-scatter grids (according to grid's material and geometrical design) were estimated. The measurements were recorded at various $\mathrm{X}$-ray tube applied voltage $(\mathrm{kVp})$ ranging from $50 \mathrm{kVp}$ to $110 \mathrm{kVp}$, and fixed exposure intensity of $5 \mathrm{mAs}$. The backscattered radiation reduction effects of different types of anti-scatter grids during radiographic imaging are illustrated in Figure 3. The backscattered doses were measured with and without the use of fabricated anti-scatter grids at different X-ray tube voltages $(\mathrm{kVp})$.

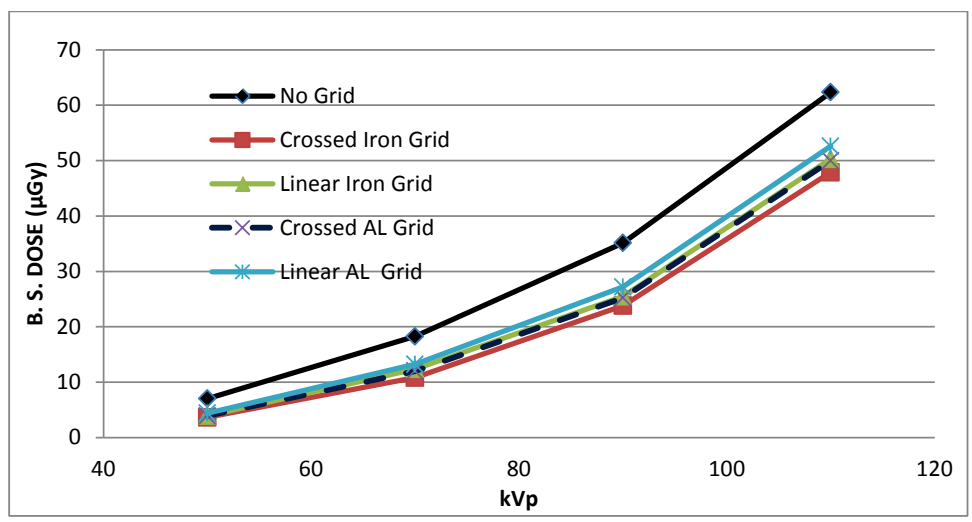

Figure 3: Backscattered radiation doses at different $\mathrm{kVp}$ for stationary $\mathrm{X}$-ray unit.

The relative dose of backscattered photons increased with increasing X-ray tube voltage $(\mathrm{kVp})$ for all setups due to the fact that more scatter interactions occur at higher photon energy. The anti-scatter grids attenuate backscattered radiation by 
absorbing and trapping the backscattering photons. These grids stop backscattered photons which do not travel parallel to the grids' strips or that incident on grid's baseplate. Thus, the reduction of backscattered radiation depends on the geometrical properties of anti-scatter grids and the direction of radiation. The disparity in the reducing capabilities of the grids can also be attributed to variations in atomic numbers and densities of the grid's material as well as grid's geometrical designs. These results are consistent with earlier findings by Hendee and Ritenour, as well as Hobbie and Roth who recommended that the grid's strips should be completely opaque to scattered radiation and should not release absorbed X-rays as scattered X-ray photons. ${ }^{12,13}$

With regards to the effect of primary photon energy, increasing the peak tube voltage $(\mathrm{kVp})$ will cause more photons with high energies to penetrate the exposed target and interact more with X-ray tables, bucky or wall. Compton effect is one of these interactions in which the scattered photons may be reflected back at different backscattering angles. Thus, the level of reduction is dependent on the incident radiation energy $(\mathrm{kVp})$. Utilising different types of anti-scatter grids presented reduction in backscattered radiation dose. The amount of backscattering dose reduction by fabricated grids depends on the incident radiation energy $(\mathrm{kVp})$ for estimating and comparing attenuation capabilities of different anti-scatter grids. The percentages of backscattered radiation reduction as a result of using grids are calculated. The capabilities of different anti-scatter grids types can be determined according to grid material and geometrical design of grid. The percentages of backscattered radiation reduction can be used to compare the effectiveness of different anti-scatter grids for removing backscattered photons.

Figure 4 illustrates the percentages of backscattered dose as a function of peak tube voltage. The radiation reduction capabilities of anti-back scatter grids were observed to increase with decreasing X-ray tube voltage and incident radiation energy. All types of anti-scatter grids presented their best capabilities in removing backscattered radiation at lowest $\mathrm{X}$-ray tube voltage of $50 \mathrm{kVp}$, and least capabilities at the highest $\mathrm{X}$-ray tube voltage of $110 \mathrm{kVp}$. 


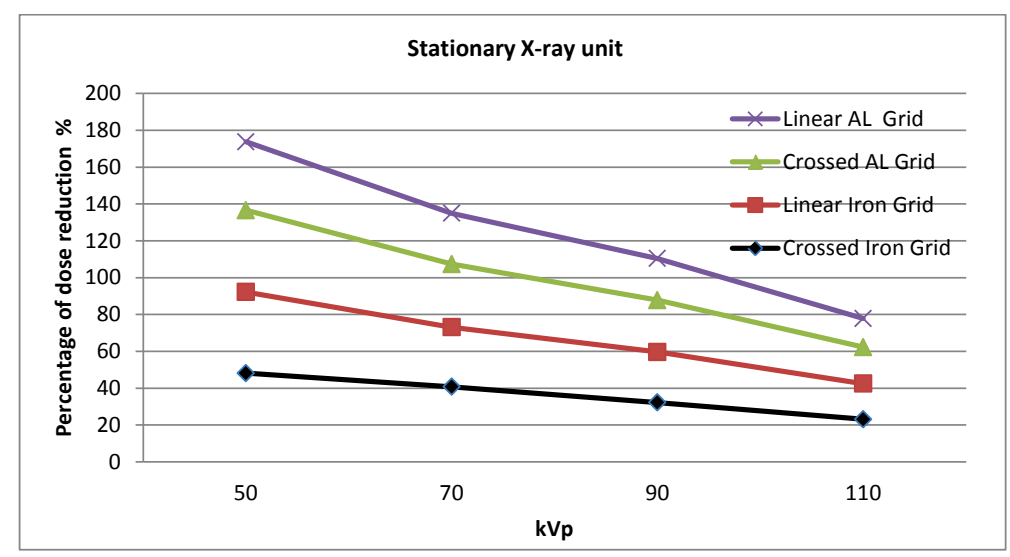

Figure 4: The effectiveness of anti-scatter grids at different tube voltage.

In terms of grid type, the crossed iron grid exhibited the best backscattered radiation dose reducing capability at different X-ray tube voltages $(\mathrm{kVp})$, particularly at lower applied voltages with percentage reduction up to $48.15 \%$. The advantage of crossed iron grid is related to the density and atomic number of the grid material and the geometrical design of grid. Whereas Abdul-Majid et al. demonstrated the possibility of reducing backscattered radiation up to about $45 \%$ by using iron panel, the variation in relative reduction percentage can be attributed to the geometrical design of fabricated crossed grid. ${ }^{7}$ Similar results have been reported by Hendee and Ritenour, who showed that crossed grids are more effective in reducing scattered radiation compared to linear grid. ${ }^{12}$ Therefore, the material and design of anti-scatter grid play an important role in determining grid effectiveness and backscattering radiation attenuating capability.

\subsection{X-ray Machine Type}

Given that X-ray spectra vary for different X-ray machines, it is advisable to determine the backscatter values for different X-ray machines. The results of stationary X-ray unit can be comparatively analysed against other X-ray sources. Procedures that have been performed for stationary X-ray units were deployed for mobile X-ray unit to evaluate the effectiveness and stability of the anti-scatter grids results. Backscattered radiation doses for the mobile X-ray unit were relatively lower compared with the stationary X-ray unit. Figure 5 shows the measured backscattered doses obtained with the mobile X-ray unit as a function of peak tube voltage $(\mathrm{kVp})$. The backscattered radiation doses increase with increasing $\mathrm{X}$-ray tube voltage. 


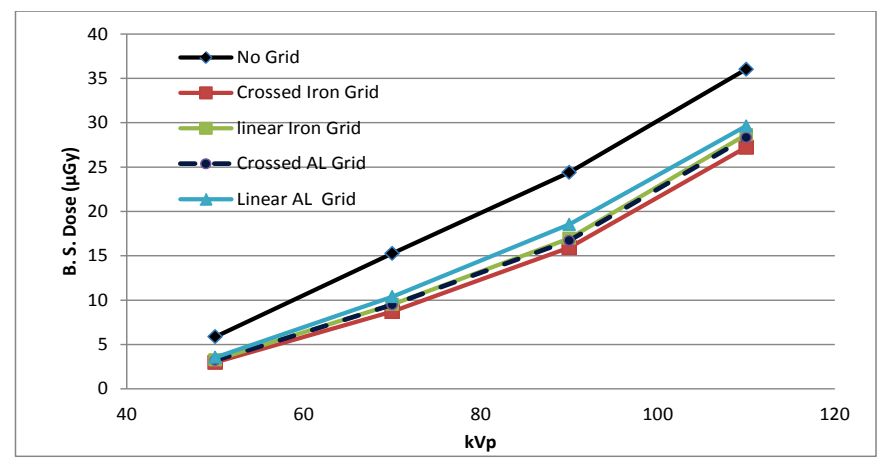

Figure 5: Backscattered radiation doses for mobile X-ray unit.

The effect of different fabricated anti-scatter grids on reducing backscattered radiation from a mobile X-ray unit was analysed. Similar to the stationary X-ray unit, all types of fabricated anti-scatter grids showed lesser backscattered radiation doses from the mobile unit at all X-ray tube voltages compared to backscattered radiation doses measured without the use of anti-scatter grid. The lowest backscattered radiation doses were obtained with the use of crossed iron grid at various $\mathrm{X}$-ray tube voltages. The percentages of backscattered radiation reduction for different anti-scatter grids at various $\mathrm{kVp}$ are calculated. Different types of anti-scatter grids proved different capabilities in attenuating backscattered radiation accoding to grid's material and geometrical design as well as the energy of primary radiation. Figure 6 shows the effectivness of several anti-scatter grids in attenuating backscattered radiation as a functon of peak tube voltage for mobile X-ray unit.

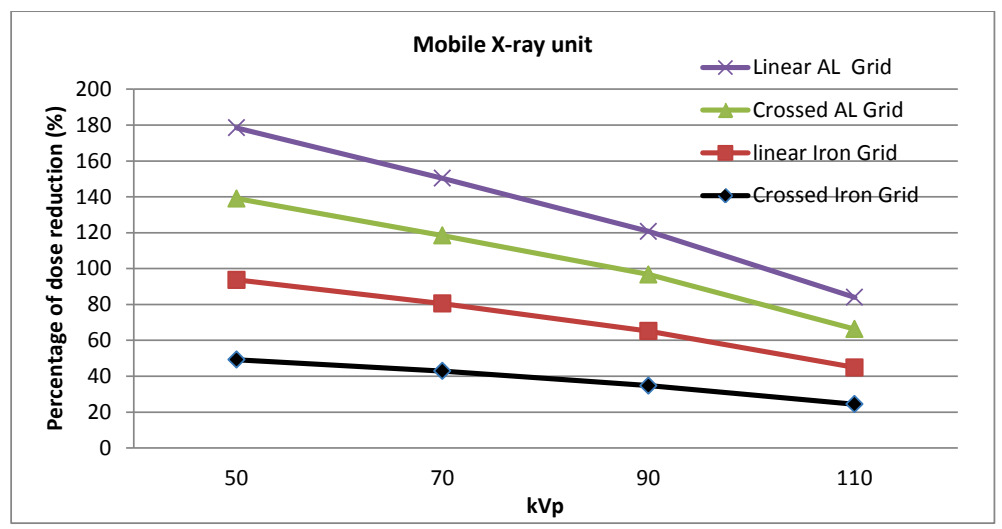

Figure 6: The effectiveness of anti-scatter grids at different $\mathrm{kVp}$. 
It can be inferred from Figure 6 that the effectiveness of different anti-scatter grids in reducing backscattered radiation from mobile units at various $\mathrm{X}$-ray tube voltages is similar to stationary X-ray units. Similarly, a clear difference was observed between capabilities of anti-scatter grid types in reducing backscattered radiation dose based on the grid material and geometrical design. Best capability in reducing backscattering radiation up to $49.24 \%$ at lower applied voltages was achieved with the use of crossed iron grid. Nonetheless, the grid's effectiveness was altered by changing the X-ray tube voltage $(\mathrm{kVp})$. High percentages in backscattered dose reduction were achieved at lower $\mathrm{X}$-ray tube voltage $(50 \mathrm{kVp})$ for all anti-scatter grid types because the lower energy photons more easily attenuated, while less percentages were achieved at high X-ray energy $(110 \mathrm{kVp})$ for the different antiscatter grids. This is consistent with the results by McGiff et al., who reported that the use of backscatter shield reduces the radiation dose that a dental operator is exposed to by up to $70 \%$ at relatively low $\mathrm{kVp} .{ }^{14}$

Collectively, the results showed that all fabricated anti-scatter grids exhibited similar effectiveness in reducing backscattered radiation for the different X-ray units (stationary and mobile). The results also indicated that there is no significant difference in the capability of each grid in attenuating the backscattered dose for both X-ray units (stationary and mobile). In addition, factors such as X-ray tube voltage $(\mathrm{kVp})$, grid material and geometrical design similarly affect the attenuation capabilities of anti-scatter grids in both X-ray units.

The contribution of this study can be summarised as follows: The reduction of backscattered radiation inside X-ray room using fabricated anti-backscatter grids can improve radiation protection for patient and workers during diagnostic examinations as well as enhance X-ray image quality. The materials utilised in this work are economical, readily available and can easily be shaped and fabricated into different designs of anti-backscatter grids. In addition, this study will offer a novel practical method to decrease backscattered radiation exposure for patients, medical personnel, public and image's receptor.

\section{CONCLUSION}

This study developed an approach to reduce backscattered radiation using fabricated anti-scatter grids. The fabricated anti-scatter grids exhibited remarkable capability in reducing backscattered radiation. The results indicated that the crossed iron and aluminium grids can be useful for decreasing backscattered radiation. The effectiveness of anti-scatter grid is dependent on the material and geometrical design of the grid. Grid material with high atomic number and mass density exhibit 
higher reduction in backscattered radiation. Yet, the crossed iron steel grid shows the best attenuation capability. In addition, the reduction of backscattered radiation dose can be obtained by adequate changes of exposure parameters such as $\mathrm{kVp}$ and $\mathrm{mAs}$.

\section{ACKNOWLEDGEMENTS}

This work was carried out in the Radiation Biophysics Laboratory, School of Physics, Universiti Sains Malaysia. The authors gratefully acknowledge the support of the school.

\section{REFERENCES}

1. Yao, W. \& Leszczynski, K. (2008). Depth distribution of multiple order X-ray scatter. Radiat. Phys. Chem., 77, 381-390, https://doi.org/10.1016/j. radphyschem.2007.12.002.

2. Wu, G. et al. (2009). Evaluation of scatter effects on image quality for breast tomosynthesis. Med. Phys., 36, 4425-4432, https://doi. org/10.1118/1.3215926.

3. Naryshkin, S. (2007). On methods for assessing radiation load on medical personnel during an endourologic intervention. Biomed. Eng., 41, 228-231, https://doi.org/10.1007/s10527-007-0053-y.

4. Bushong, S. C. (2013). Radiologic science for technologists: Physics, biology, and protection, 10th ed. New York: Elsevier.

5. Nicholas, J. (2006). Principle of patient radiation protection. Retrieved from http://www. ceessential.net on 4 Mar 2012.

6. Mohamed, A. H. (2007). Determination of scattered radiation to the testis during PA chest X-ray procedures. Project report for Distance Education, Universiti Sains Malaysia, Pulau Pinang, Malaysia.

7. Abdul-Majid, S. et al. (2005). Reduction of backscattered radiation in enclosure X-ray radiography. Paper presented at 3rd Middle East Nondestructive Testing Conference and Exhibition, 27-30.

8. Stecker, M. S. etal. (2009). Guidelines for patient radiation dose management. J. Vasc. Interv. Radiol., 20, 263-273.

9. Aichinger, $\mathrm{H}$. et al. (2011). Radiation exposure and image quality in X-ray diagnostic radiology: Physical principles and clinical applications, 2 nd ed. New York: Springer Science \& Business Media, https://doi.org/10.1016/j. jvir.2009.04.037. 
10. Jaenisch, G. R., Ewert, U. \& Jechow, M. (2010). Scatter radiation in radiography. Berlin: Federal Institute for Materials Research and Testing.

11. Neugschandtner, K. et al. (2015). Evaluation of radiation exposure in interventional radiology (IR) using active personal dosimeters (APD). Paper presented at Radiation Protection and Health Proceedings, Radiation Protection and Dosimetry, IAEA.

12. Hendee, W. R. \& Ritenour, E. R. (2003). Medical imaging physics. New York: John Wiley \& Sons.

13. Hobbie, R. \& Roth, B. J. (2007). Intermediate physics for medicine and biology, 4th ed. New York: Springer Science \& Business Media.

14. McGiff, T. J., Danforth, R. A. \& Herschaft, E. E. (2012). Maintaining radiation exposures as low as reasonably achievable (ALARA) for dental personnel operating portable hand-held X-ray equipment. Health Phys., 103, 179-185, https://doi.org/10.1097/HP.0b013e318259fa29. 